\title{
MicroRNA-27a inhibits trophoblast cell migration and invasion by targeting SMAD2: Potential role in preeclampsia
}

\author{
WENFEI ZHENG ${ }^{1,2}$, AIHUA CHEN ${ }^{2}$, HUAIJIE YANG ${ }^{2}$ and LI HONG $^{1}$ \\ ${ }^{1}$ Department of Gynecology and Obstetrics, Renmin Hospital of Wuhan University, Wuhan, Hubei 430060; \\ ${ }^{2}$ Department of Gynecology and Obstetrics, The People's Hospital of China Three Gorges University, \\ Yichang, Hubei 443000, P.R. China
}

Received March 24, 2019; Accepted January 23, 2020

DOI: $10.3892 / \mathrm{etm} .2020 .8924$

\begin{abstract}
Preeclampsia (PE) is a severe idiopathic obstetric complication that occurs worldwide. Insufficient trophoblast invasion is a characteristic of the pathogenesis of PE. MicroRNA-27a (miR-27a) has been reported to be highly expressed in PE placentas. The aim of the present study was to investigate the role and underlying mechanisms of miR-27a in the pathogenesis of PE. The expression level of miR-27a was evaluated in the placenta and serum from patients with $\mathrm{PE}$ and healthy pregnant women. Cell Counting Kit-8 and flow cytometry assays were performed to detect human HTR-8/SVneo trophoblast proliferation and apoptosis after miR-27a overexpression or inhibition. In addition, Transwell assays were used to measure cell migration and invasion. A luciferase reporter assay was performed to determine the interaction between miR-27a and SMAD2. The present results suggested that miR-27a expression level was significantly increased in PE placentas and serum. In addition, miR-27a overexpression suppressed cell migratory and invasive abilities, impaired proliferation and promoted apoptosis in human trophoblasts. It was demonstrated that miR-27a may target SMAD and contribute to trophoblast invasion. Collectively, the results of the present study suggested that miR-27a inhibited trophoblast cell migration and invasion by targeting SMAD2, thus presenting a promising therapeutic target for PE.
\end{abstract}

\section{Introduction}

Preeclampsia (PE) is a severe idiopathic obstetric complication that occurs during pregnancy (1). PE affects $\sim 3 \%$ of pregnant women worldwide (2). PE has many contributing factors and a multi-pathway pathogenesis (3), however the specific

Correspondence to: Dr Li Hong, Department of Gynecology and Obstetrics, Renmin Hospital of Wuhan University, 99 Zhangzhidong Road, Wuhan, Hubei 430060, P.R. China

E-mail: v220676@163.com

Key words: preeclampsia, microRNA, SMAD2, transforming growth factor pathogenesis of this condition is not fully understood. The main factors causing PE include superficial implantation of the placenta, insufficient placental perfusion, placental hypoxia and ischemia, oxidative stress and immune imbalance (4). A functional placenta is important for maintaining pregnancy and fetal development (5). At the start of a pregnancy, extravillous trophoblasts invade the decidua and inner myometrium of the mother, a process which is required for the progression of the pregnancy $(6,7)$.

Transforming growth factor- $\beta 1$ (TGF- $\beta 1$ ) is a well-known pleiotropic cytokine. TGF- $\beta 1$ can induce a number of biological functions, including embryogenesis, tissue morphogenesis of the embryo, cell proliferation, migration, differentiation and immune responses (8-11). The role of TGF- $\beta 1$ in different biological functions is determined by the context in the body and interactions with other signaling pathways (12). It has been reported that TGF- $\beta 1$ serves oncogenic roles in malignant tumors, such as in gastrointestinal, breast and liver cancer $(13,14)$. TGF- $\beta 1$ is also involved in the invasion of trophoblasts $(15,16)$, as well as placenta progenitor cell differentiation into trophoblasts (17). The canonical pathway, in which TGF- $\beta 1$ regulates cell invasion and migration, is mediated by SMAD $(18,19)$. SMADs are intracellular proteins that transduce extracellular signals from TGF- $\beta$ ligands to the nucleus, where they activate downstream gene transcription (20). SMAD2 was demonstrated to serve critical roles in the migration and invasion of cancer cells (21-23).

MicroRNAs (miRNAs/miRs) are small, single-stranded non-coding RNAs that serve a variety of roles in a series of disorders, including in malignant tumors and PE, via post-translational modifications $(24,25)$. A previous study measured cell-free miRNAs in plasma from patients with prostate cancer and healthy donors, and demonstrated that miR-141 expression levels were high in patients with advanced stage cancer and associated with the Gleason score value (26). Peng et al (27) reported that miR-429 acted as a tumor suppressor gene by inhibiting astrocytoma proliferation and invasion. Additionally, miRNAs have been indicated to serve critical roles in the pathogenesis of PE (28). In a previous study, miR-29b suppressed trophoblast cell invasion and promoted apoptosis in patients with PE (29). miR-30a-3p was overexpressed in the placenta of women with $\mathrm{PE}$, and was revealed to induce HTR-8/SVneo cell apoptosis while inhibiting the 
invasive capacity of JEG-3 cells (30). The overexpression of microRNA-376c promotes the proliferation, migration and invasion of trophoblasts, and the growth of placental explants by inhibiting TGF- $\beta$ and Nodal signaling (31). miR-27a has also been reported to serve oncogenic roles in a variety of malignant tumors (32). In lung cancer, miR-27a is significantly overexpressed and is indicated to stimulate cancer cell proliferation and invasion by targeting SMAD2 and SMAD4 (21). In addition, the miR-27a/miR-27a complex was demonstrated to contribute to the metastasis of osteosarcoma (32). A previous microarray study indicated that multiple miRNAs, including $\mathrm{miR}-27 \mathrm{a} / \mathrm{b}$, regulate the onset of PE (33). Moreover, miR-27a has been shown to be significantly upregulated in the plasma and placenta of patients with PE (34). However, to the best of our knowledge, the mechanisms via which miR-27a regulates PE have not yet been elucidated.

The present study investigated the expression levels of miR-27a in plasma and placentas from patients with PE. To identify the molecular mechanisms underlying miR-27a regulation of trophoblast function, the present study investigated cell functions after overexpressing or inhibiting miR-27a. Additionally, the present study predicted the potential target of miR-27a using miRNA target prediction databases. The present results may facilitate the development of novel diagnostic and latent targeted therapies for PE.

\section{Materials and methods}

Sample collection and cell culture. A total of 35 pregnant women with severe PE and 20 healthy pregnant women who underwent caesarean section at the Department of Obstetrics and Gynecology of Renmin Hospital of Wuhan University from May 2015 to June 2016 were recruited for the current study. The diagnostic criteria for PE followed that of the American College of Obstetricians and Gynecologists, with either severe hypertension ( $\geq 160 \mathrm{mmHg}$ and/or $110 \mathrm{mmHg}$ ) plus mild proteinuria or mild hypertension plus severe proteinuria ( $>2 \mathrm{~g} / 24 \mathrm{~h}$ or $>2+$ ) (29). Other recruitment criteria for patients in the two groups included singleton pregnancies and no other complications, including premature membrane rupture, cardiac or renal disease, hypertension history and maternal infection. The clinical features of all patients are presented in Table I. Placenta tissues (five sites) were collected from different placental lobules and stored at $-80^{\circ} \mathrm{C}$. Peripheral blood samples $(5 \mathrm{ml})$ from patients in the two groups were collected at delivery. The current study was approved by The Ethics Committee of Renmin Hospital of Wuhan University, and written informed consent was obtained from all patients.

HTR-8/SVneo cells, a human trophoblast cell line, were purchased from the American Type Culture Collection and cultured in RPMI 1640 medium (Sigma-Aldrich; Merck KGaA) supplemented with $10 \%$ FBS (Gibco; Thermo Fisher Scientific, Inc.) and 1\% penicillin (New Cell and Molecular Biotech Co., Ltd.) and 1\% streptomycin (New Cell and Molecular Biotech Co., $\mathrm{Ltd})$ at $37^{\circ} \mathrm{C}$ with $5 \% \mathrm{CO}_{2}$.

Cell transfection. miR-27a inhibitor and mimic, and the corresponding negative controls (Shanghai GenePharma Co. Ltd.) were transfected into HTR-8/SVneo cells at a concentration of $2.5 \mu \mathrm{g} /$ well using Lipofectamine $3000^{\circledR}$ reagent
(Invitrogen; Thermo Fisher Scientific, Inc.) according to the manufacturer's protocols. The sequences were as follows (5'-3'): miR-27a mimic, UUCACAGUGGCUAAGUUCCGC; mimic NC, UUGUACUACACAAAAGUACUG; inhibitor, GCGGAACUUAGCCACUGUGAA; inhibitor NC, CAGUAC UUUUGUGUAGUACAA. In addition, SMAD2 overexpression plasmid and negative control (Shanghai GenePharma Co., Ltd.) were transfected into HTR-8/SVneo cells with Lipofectamine ${ }^{\circledR} 3000$ reagent (Invitrogen; Thermo Fisher Scientific, Inc.). HTR-8/SVneo cells were seeded into six-well plates at a density of $60-70 \%$. After adhesion, miR-27a inhibitor $(2.5 \mu \mathrm{g} / \mathrm{well})$ and mimic $(2.5 \mu \mathrm{g} / \mathrm{well})$, or SMAD2 overexpression plasmid $(2.5 \mu \mathrm{g} / \mathrm{well})$ and the corresponding negative controls $(2.5 \mu \mathrm{g} /$ well $)$ were transfected into cells using Lipofectamine $3000^{\circledR}$ reagent. A period of $6 \mathrm{~h}$ later, the culture medium with the transfection reagent was changed to RPMI 1640 medium (Sigma-Aldrich; Merck KGaA) supplemented with $10 \%$ FBS (Gibco; Thermo Fisher Scientific, Inc.) and $1 \%$ penicillin (NCM Biotech, China) and $1 \%$ streptomycin (NCM Biotech, China). After a period of $48 \mathrm{~h}$, the transfection efficiency was detected and confirmed. Cells were harvested for further experiments after $48 \mathrm{~h}$ of transfection.

Reverse transcription-quantitative $(R T-q) P C R$. Total RNA was isolated from placentas and cell lines using a Takara RNA extraction kit (Takara Bio, Inc.) and quantified using a spectrophotometer (BioTek, US). cDNA was synthesized from miRNA using a SuperScript II kit (Invitrogen; Thermo Fisher Scientific, Inc), and cDNA was reverse transcribed from mRNA using a PrimeScript RT reagent kit with gDNA Eraser (Takara Bio, Inc.). The RT temperature protocol is as follows: $37^{\circ} \mathrm{C}$ for $15 \mathrm{~min}, 85^{\circ} \mathrm{C}$ for $5 \mathrm{sec}$ and $4^{\circ} \mathrm{C}$ for $10 \mathrm{~min}$. PCR was performed using a SYBR Premix Ex Taq II kit (Takara Bio, Inc.). The following thermocycling conditions were used for the qPCR: Initial denaturation at $95^{\circ} \mathrm{C}$ for $30 \mathrm{sec}$; 40 cycles of denaturation at $95^{\circ} \mathrm{C}$ for $5 \mathrm{sec}$, primer annealing and extension at $60^{\circ} \mathrm{C}$ for $34 \mathrm{sec}$; and melting curve analysis denaturation at $95^{\circ} \mathrm{C}$ for $15 \mathrm{sec}$, primer annealing at $60^{\circ} \mathrm{C}$ for $1 \mathrm{~min}$ and denaturation at $95^{\circ} \mathrm{C}$ for $15 \mathrm{sec}$. U6 and GAPDH were used as internal controls for the quantification of miRNA and mRNA, respectively. The following primers were used: miR-27a forward, 5'-ACAGGC TAGCGCCGCCTAAC-3' and reverse, 5'-CCTTAAGGCCCA AGATTACG-3'; SMAD2 forward, 5'-TGAGTGTGGGATTT GACCAG-3' and reverse, 5'-TGTGTTTGGAGTGGGTTT CA-3'; GAPDH forward, 5'-TCCACTGGCGTCTTCACC-3' and reverse, 5'-GGCAGAGATGATGACCCTTTT-3'; and U6 forward, 5'-TGCGGGTGCTCGCTTCGCAGC-3' and reverse, 5'-CCAGTGCAGGGTCCGAGGT-3'. Each assay was performed in duplicate with triplicate samples, and expression levels were calculated using the $2^{-\Delta \Delta \mathrm{Cq}}$ method (35).

Transwell assay. Transwell assay chambers were purchased from Corning, Inc. Transfected HTR-8/SVneo cells were seeded into the upper chambers at a density of $4 \times 10^{4}$ cells per well with (for invasion assays) or without Matrigel (for migration assays) and without FBS. RPMI 1640 medium (Gibco; Thermo Fisher Scientific, Inc.) with $20 \%$ FBS was added to the lower chambers. Cells were then incubated at $37^{\circ} \mathrm{C}$ with $5 \% \mathrm{CO}_{2}$. After $48 \mathrm{~h}$, cells in the upper chambers were wiped away using cotton swabs, and cells in the lower chambers 
Table I. Clinicopathological characteristics of PE and healthy pregnant women.

\begin{tabular}{lccc}
\hline Clinicopathological features & Healthy control $(\mathrm{n}=20)$ & Patients with PE $(\mathrm{n}=35)$ & P-value \\
\hline Maternal age, years & $27.3 \pm 1.9$ & $28.2 \pm 2.1$ & $>0.05$ \\
Gestational age, weeks & $40 \pm 2.1$ & $36.6 \pm 3.4$ & $<0.05$ \\
Placental weight, g & $462 \pm 56.3$ & $425.8 \pm 50.2$ & $<0.05$ \\
Systolic blood pressure, $\mathrm{mmHg}$ & $104.5 \pm 12.4$ & $157.5 \pm 15.3$ & $<0.05$ \\
Diastolic blood pressure, $\mathrm{mmHg}$ & $79.3 \pm 7.8$ & $121.5 \pm 6.4$ & $<0.05$ \\
Infant birth weight, g & $3563.4 \pm 234.2$ & $2783.4 \pm 312.2$ & $<0.05$ \\
Sex of infants & & 16 & $>0.05$ \\
Male & 8 & 19 & $3.51 \pm 0.98$ \\
Female & 12 & & $<0.05$ \\
Proteinuria, $\mathrm{g} / 24 \mathrm{~h}$ & None & & \\
\hline
\end{tabular}

PE, Preeclampsia.

were fixed in $4 \%$ paraformaldehyde at room temperature for $15 \mathrm{~min}$ and stained using a $0.1 \%$ crystal violet solution at room temperature for $10 \mathrm{~min}$. The migrated or invaded cells in five random fields were counted and photographed under a light microscope (magnification, x200).

Western blot analysis. Total protein was extracted from cells using RIPA lysis buffer (Beyotime Institute of Biotechnology). Cells were incubated with RIPA lysis buffer for $20 \mathrm{~min}$ on ice and centrifuged at $4^{\circ} \mathrm{C}$ for $16,099 \mathrm{x}$ g for $30 \mathrm{~min}$. The supernatants were removed and the protein concentrations were determined using a BCA kit (Beyotime Institute of Biotechnology). Then, $40 \mu \mathrm{g}$ of denatured protein was separated on $10 \%$ SDS-PAGE gels and transferred onto PVDF membranes. After blocking in $5 \%$ non-fat milk at room temperature for $1 \mathrm{~h}$, the membranes were incubated with primary antibodies at $4^{\circ} \mathrm{C}$ overnight. The primary antibodies used were monoclonal rabbit anti-Smad2 (cat. no. ab33875), anti-Bax (cat. no. ab32503) and anti-Bcl-xl (cat. no. ab32370) (all 1:1,000; Abcam). A GAPDH antibody (1:1,000; cat. no. ab181602; Abcam) was used as a control for normalizing protein expression. On the second day, the membranes were incubated with secondary antibody (goat anti-rabbit immunoglobulin G H\&L (HRP) $(1: 10,000$; cat. no. ab97051; Abcam) at room temperature for $1 \mathrm{~h}$ and washed three times with 1x PBS-Tween-20 for $10 \mathrm{~min}$. Bands were detected using a chemiluminescence kit (Sigma-Aldrich; Merck KGaA). The band intensities were analyzed using ImageJ 1.51j8 (National Institutes of Health).

Cell Counting Kit-8 (CCK-8) assay. CCK-8 was performed according to the manufacturer's protocol. Transfected HTR-8/SVneo cells were seeded in 96-well plates at a density of $5 \times 10^{3}$ cells/well. After 48 h, $100 \mu \mathrm{l}$ of RPMI 1640 medium and $10 \mu \mathrm{l}$ of CCK-8 reagent (Dojindo Molecular Technologies, Inc.) were added to each well and incubated at $37^{\circ} \mathrm{C}$ for $40 \mathrm{~min}$. The relative proliferation ability was measured at a wavelength of $450 \mathrm{~nm}$.

Cell apoptosis assay. The apoptotic rate of cells was detected using an Annexin V-FITC Apoptosis Detection kit (Becton,
Dickinson and Company) following the manufacturer's protocol. Transfected HTR-8/SVneo cells were trypsinized and washed with cold PBS three times. The resuspended cells were stained with $5 \mu \mathrm{l}$ Annexin V-FITC and $5 \mu \mathrm{l} \mathrm{PI}$ at room temperature for $5 \mathrm{~min}$ in the dark. The apoptotic rate was analyzed using a flow cytometer (FlowJo 7.6; Becton, Dickinson and Company).

Luciferase reporter assay. The wild-type 3'untranslated region (UTR) of SMAD2 and a mutated (MUT) 3'UTR containing the predicted binding sites of miR-27a were cloned into the pGL3 vector (Promega Corporation). The binding sites were predicted using TargetScan (http://www.targetscan.org/vert_72/) and miRanda (http://www.microrna.org/). The reporters containing mutant (MUT) or wild-type (WT) SMAD2 were transfected into trophoblast cell lines along with a miR-27a mimic, inhibitor or negative control with Lipofectamine ${ }^{\circledR} 3000$ (Invitrogen; Thermo Fisher Scientific, Inc.). After 48 h, luciferase activity was detected using a Dual-Luciferase ${ }^{\circledR}$ Reporter Assay System kit (Promega Corporation). Renilla luciferase activity was used as a normalization control.

Statistical analysis. SPSS 16.0 software (SPSS, Inc.) was used to perform statistical analyses. Data are presented as the mean \pm standard deviation, and experiments were repeated $\geq 3$ times. Independent Student's t-test was performed to compare the differences of measurement data between two groups and $\chi^{2}$-test was performed to compare the differences of categorical data. In addition, a one-way ANOVA followed by Tukey test was performed to compare the differences among multiple groups. $\mathrm{P}<0.05$ was considered to indicate a statistically significant difference.

\section{Results}

miR-27a is significantly upregulated in PE placentas and serum. To investigate the potential roles of miR-27a in PE, PE and healthy control clinical samples were collected and the expression levels of miR-27a was detected using RT-qPCR. The present results indicated that miR-27a expression levels 
A

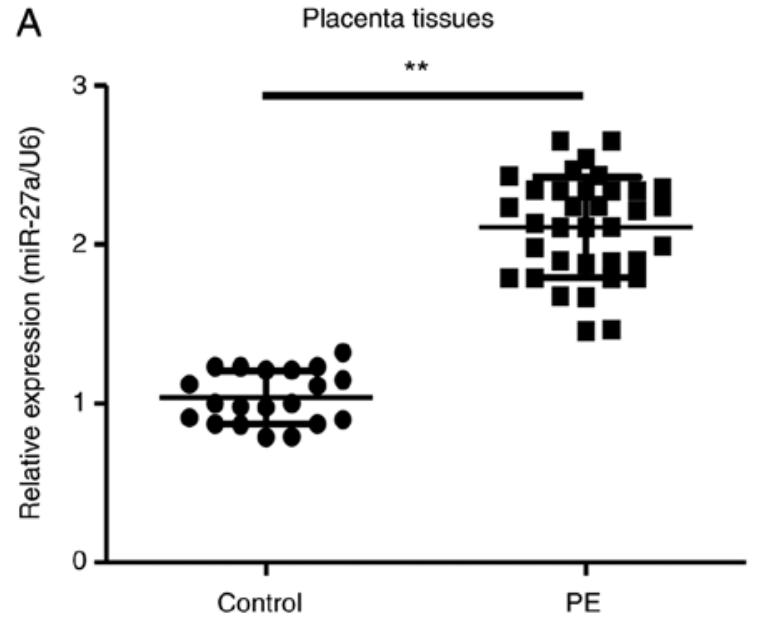

B

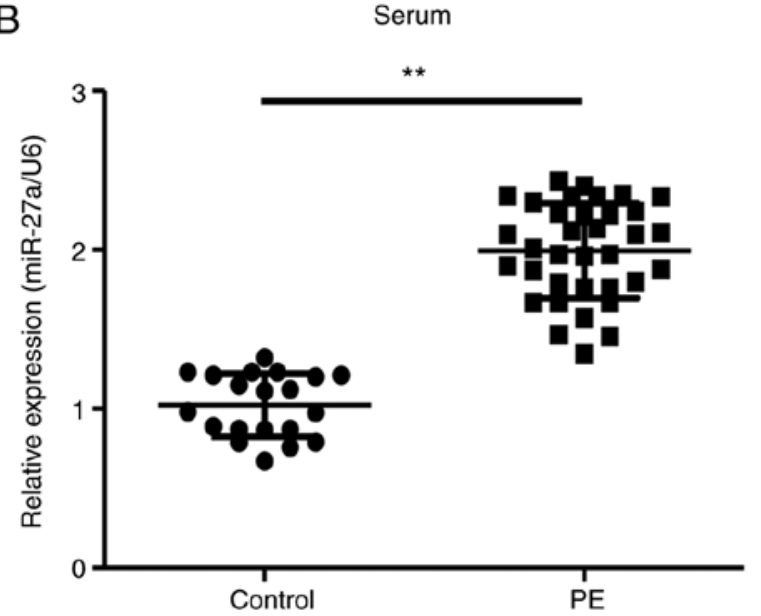

Figure 1. miR-27a is upregulated in PE placenta and serum. Reverse transcription-quantitative PCR was performed to detect miR-27a expression level in (A) placenta tissues and (B) serum of pregnant women with or without PE. ${ }^{* *} \mathrm{P}<0.01$. PE, preeclampsia; miR-27a, microRNA-27a.

were significantly increased in the placenta and serum from pregnant women with PE compared with healthy pregnant women (Fig. 1). Therefore, miR-27a may serve a critical role in PE pathogenesis.

Overexpression of miR-27a suppresses the migration and invasion of human trophoblasts. To further investigate the role of miR-27a in PE development, miR-27a mimic, inhibitor or negative controls were transfected into trophoblasts. The transfection efficiency was verified using RT-qPCR, with the mimic and inhibitor increasing and decreasing the expression level of miR-27a, respectively, compared with the corresponding controls (Fig. 2A). The present study also examined the migratory and invasive abilities of HTR-8/SVneo cells after miR-27a mimic or inhibitor transfection. The migratory and invasive abilities were significantly inhibited after miR-27a overexpression, while miR-27a inhibition improved the migratory and invasive abilities of HTR-8/SVneo cells (Fig. 2B and C). Collectively, the present results suggested that the ectopic expression of miR-27a impaired the migratory and invasive abilities of human trophoblasts, and may contribute to the pathogenesis of PE.

Overexpression of $m i R-27 a$ impairs cell proliferation and promotes apoptosis in human trophoblasts. To investigate the effects of miR-27a expression level on the proliferation of HTR-8/SVneo cells, CCK-8 and cell apoptosis assays were performed after miR-27a overexpression or inhibition. Additionally, apoptotic-related proteins were analyzed using western blot analysis. The results demonstrated that the overexpression of miR-27a impaired proliferation and promoted apoptosis in human trophoblasts (Fig. 3A and B). The pro-apoptotic protein Bax was upregulated, while the anti-apoptotic protein Bcl-xl was downregulated following miR-27a mimic transfection (Fig. 3C). Therefore, miR-27a deficiency may stimulate cell proliferation and suppress apoptosis in trophoblasts in PE.

miR-27a targets SMAD2. To further elucidate the mechanisms by which miR-27a overexpression regulates the migration and invasion of trophoblasts, a number of databases (miRanda and TargetScan) were used to predict the possible targets of miR-27a. The bioinformatic prediction results indicated that SMAD2 was a target of miR-27a, with a binding site in the 3'UTR (Fig. 4A). To investigate whether miR-27a regulated the invasion of HTR-8/SVneo cells by targeting SMAD2, WT and MUT luciferase reporters containing the 3'UTR of SMAD2 were constructed. After co-transfection with miR-27a mimic or inhibitor, the luciferase activity was measured. The results suggested that in the WT group, miR-27a overexpression decreased the luciferase activity of cells compared with the miR-27a negative control, while miR-27a downregulation increased luciferase activity (Fig. 4B). However, no changes were identified in the MUT 3'UTR groups compared with their corresponding controls (Fig. 4B). Additionally, the mRNA and protein levels of SMAD2 were significantly decreased in the miR-27a-overexpressing cells, but increased in the miR-27a-deficient cells (Fig. 4C and D).

Overexpression of SMAD2 stimulates the invasion of HTR-8/SVneo cells and reverses the miR-27a upregulationmediated inhibition of cell invasion. Cell invasion was detected following transfection with a SMAD2 overexpression plasmid or negative control (Fig. 5A). The results revealed that HTR-8/SVneo cell invasion was increased by SMAD2 overexpression compared with the negative control group (Fig. 5B). To further demonstrate that miR-27a suppressed HTR-8/SVneo cell invasion by targeting SMAD2, the SMAD2 overexpression plasmid was transfected into miR-27a-overexpressing cells and confirmed using RT-qPCR (Fig. 5C). Transwell assay results indicated that cell invasion inhibition that was caused by miR-27a overexpression was reversed by SMAD2 overexpression (Fig. 5D). Collectively, the present results suggested that miR-27a may regulate the invasion of human trophoblasts, at least partially by targeting SMAD2.

\section{Discussion}

The present results suggested that miR-27a expression level was significantly higher in placenta and blood samples from pregnant women with PE compared with healthy pregnant 
A

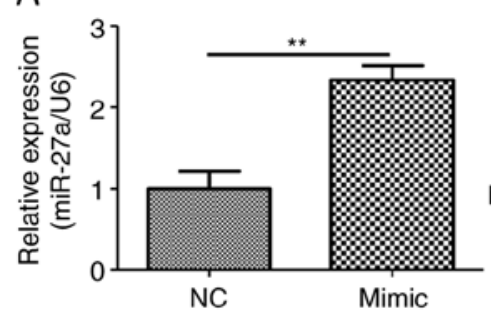

B
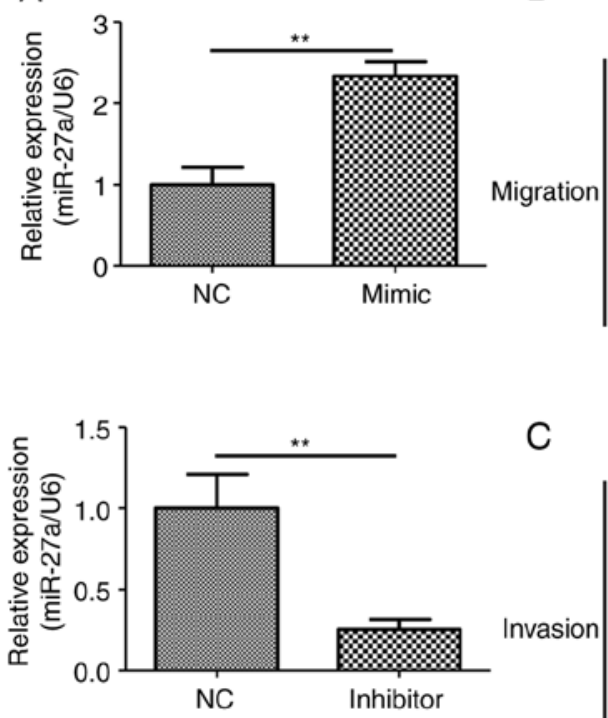

C

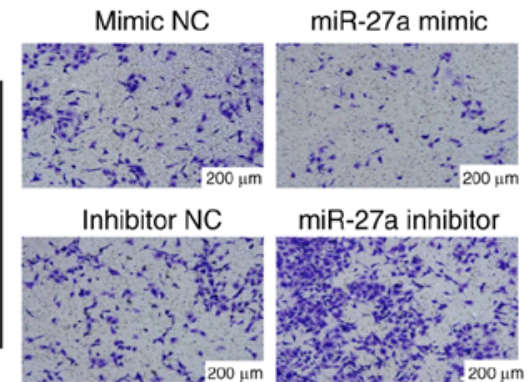

Mimic NC

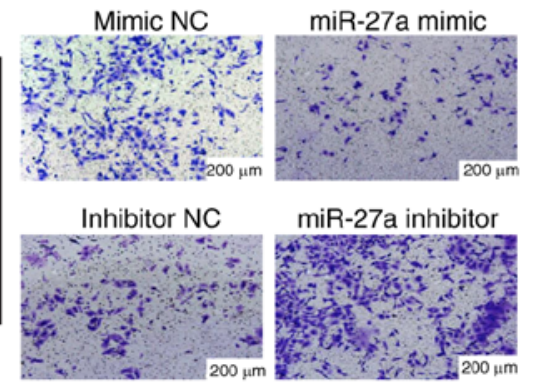

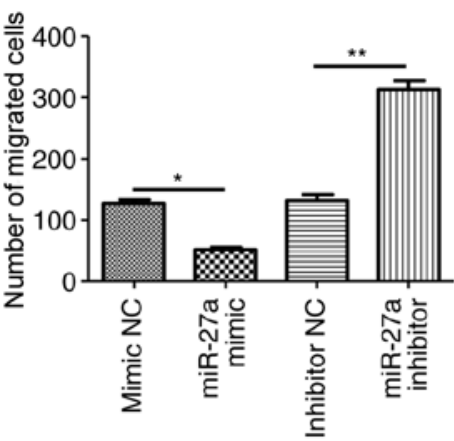

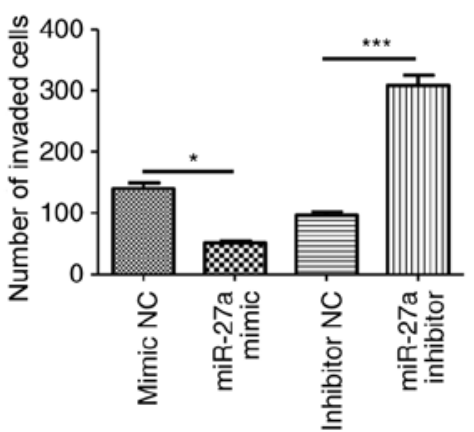

Figure 2. Overexpression of miR-27a suppresses the migratory and invasive abilities of human trophoblasts. (A) Reverse transcription-quantitative PCR was performed to measure the transfection effect of miR-27a mimic and inhibitor. HTR-8/SVneo cells were transfected with miR-27a mimic, miR-27a inhibitor or the corresponding NCs. Transwell assay was performed to measure the (B) migration and (C) invasion of trophoblast. Scale bar, $200 \mu \mathrm{m}$. ${ }^{*} \mathrm{P}<0.05,{ }^{* *} \mathrm{P}<0.01$, ${ }^{* * *} \mathrm{P}<0.001$. miR-27a, microRNA-27a; NC, negative control.

A

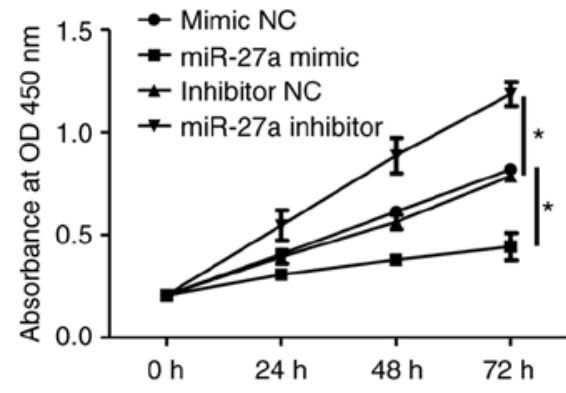

B
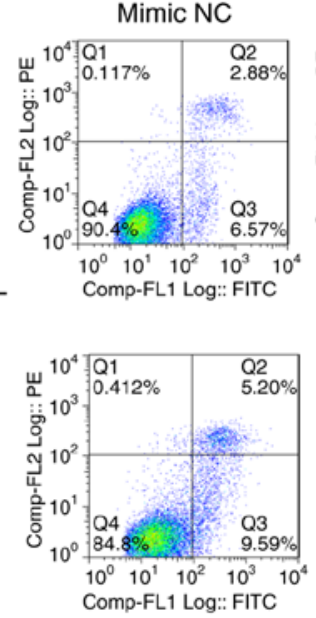

Inhibitor NC
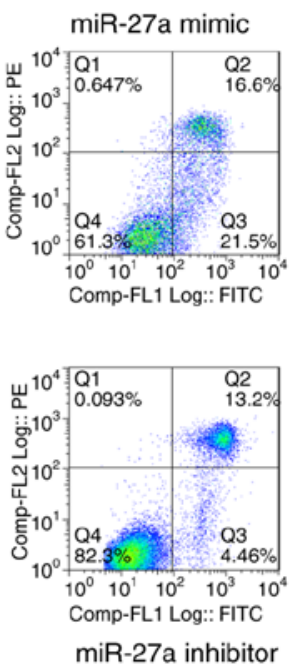

miR-27a inhibitor

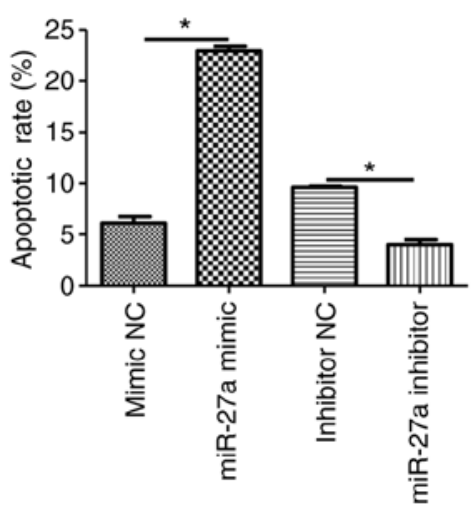

GAPDH

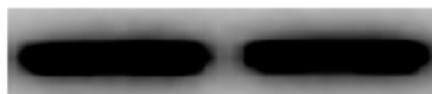

Figure 3. Overexpression of miR-27a impairs the proliferation and promotes the apoptosis of human trophoblasts. HTR-8/SVneo cells were transfected with miR-27a mimic, miR-27a inhibitor or the corresponding NCs. (A) Cell Counting Kit-8 was used to detect cell proliferation at 24, 48 and $72 \mathrm{~h}$. (B) Flow cytometry was performed to assess the cell apoptotic rate at $48 \mathrm{~h}$ after transfection. (C) Western blot analysis was used to detect pro-apoptotic and anti-apoptotic protein at $48 \mathrm{~h}$ after transfection. GAPDH was used as an internal control. "P<0.05. miR-27a, microRNA-27a; NC, negative control; OD, optical density.

women. The overexpression of miR-27a impaired the proliferative ability and induced apoptosis of human trophoblasts. In addition, the migratory and invasive capacities of trophoblasts were significantly reduced after miR-27a overexpression. To investigate the underlying mechanisms governing this, bioinformatics analysis was performed to identify potential targets of miR-27a, and SMAD2 was predicted as a direct target of miR-27a. Luciferase reporter assays, RT-qPCR, western blot analysis and rescue experiments were used to demonstrate that miR-27a regulated HTR-8/SVneo invasion, at least in part, by targeting SMAD2. Therefore, miR-27a may be a promising therapeutic target for PE. 


\section{A}

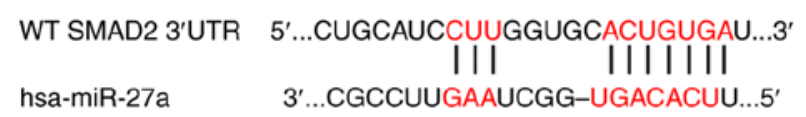

MUT SMAD2 3'UTR $5{ }^{\prime}, .$. CUGCAUCUGGGGUGCTGUCACAGU....3'
B

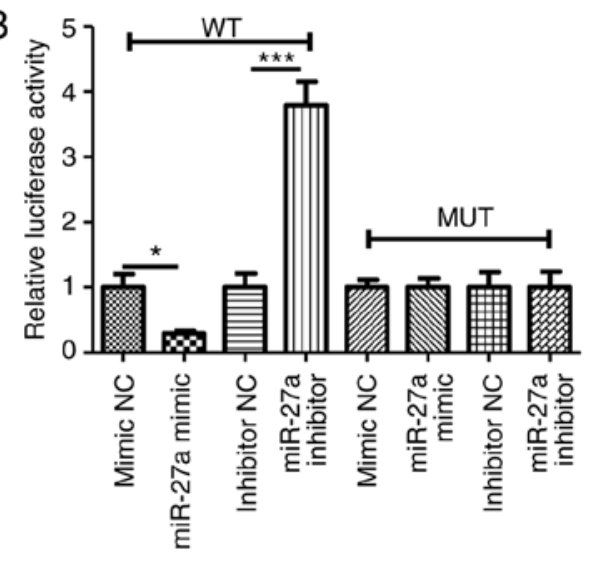

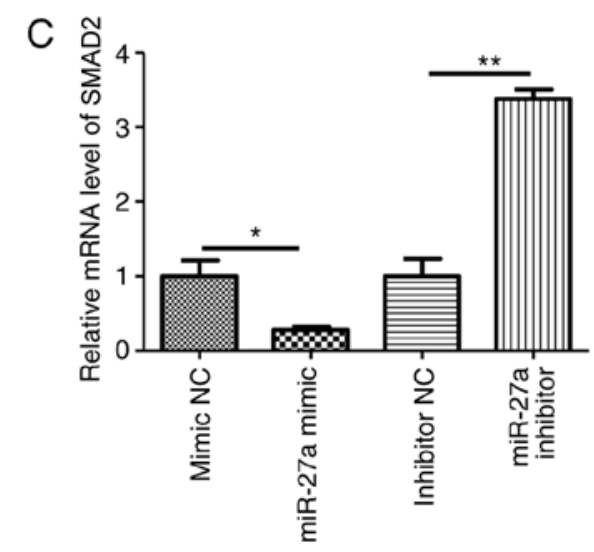

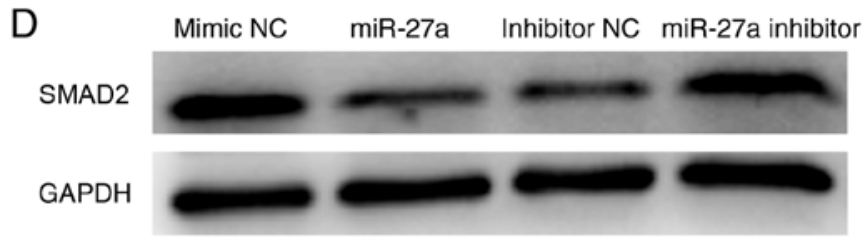

Figure 4. SMAD2 is a direct target of miR-27a. (A) Putative binding site of miR-27a and SMAD2. (B) Luciferase assay of HTR-8/SVneo cells co-transfected with firefly luciferase constructs containing the SMAD2 WT or MUT 3'-UTRs, and miR-27a mimics, inhibitor or the corresponding negative controls. (C) mRNA and (D) protein expression level of SMAD2 after transfection with miR-27a mimic or inhibitor was measured by reverse transcription-quantitative PCR and western blotting. ${ }^{*} \mathrm{P}<0.05,{ }^{* *} \mathrm{P}<0.01,{ }^{* * *} \mathrm{P}<0.001$. WT, wild-type; MUT, mutant; 3'UTR, 3'untranslated regions; miR-27a, microRNA-27a; NC, negative control.
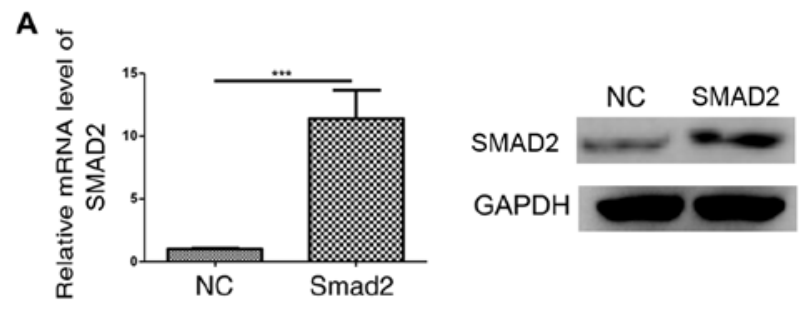

B
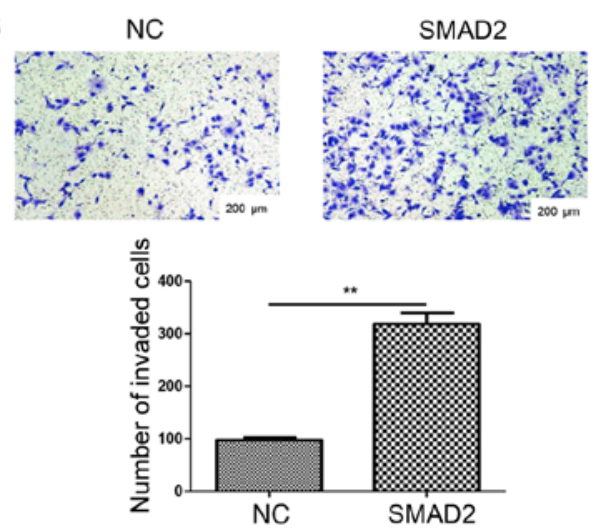

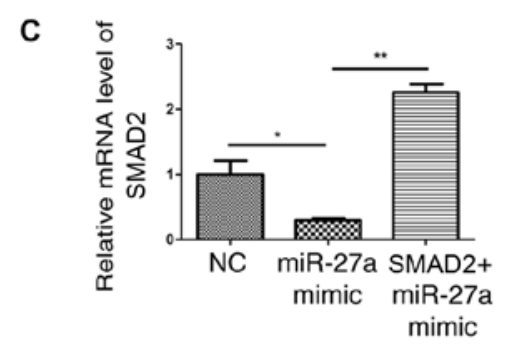

D

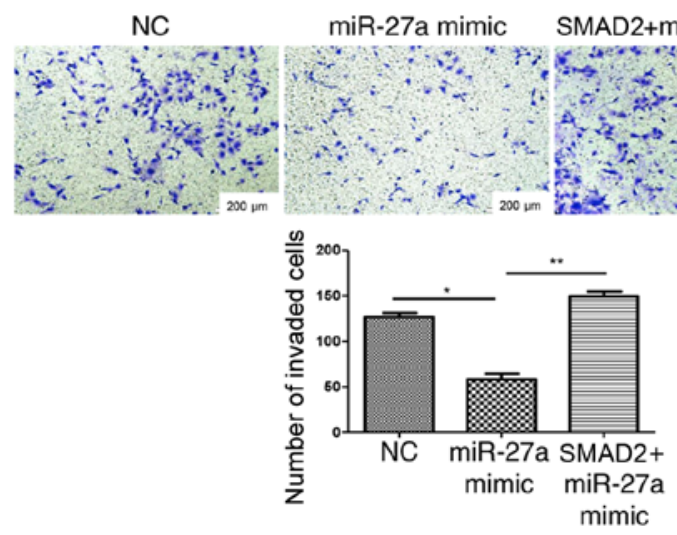

Figure 5. Overexpression of SMAD 2 stimulates the invasion of HTR-8/SVneo and reverses miR-27a upregulation-mediated inhibition effects on cell invasion. (A) SMAD2 overexpression was confirmed by RT-qPCR and western blotting. (B) HTR-8/SVneo cells were transfected with SMAD2 overexpression plasmid or negative controls. Transwell assay was used to detect invasion capacity. (C) RT-qPCR was performed to demonstrate the rescue effect of SMAD2 overexpression after miR-27a mimic transfection. (D) HTR-8/SVneo cells were transfected with miR-27a mimic, SMAD2 overexpression plasmid or negative control. Transwell assay was used to detect cell invasion. ${ }^{*} \mathrm{P}<0.05,{ }^{* *} \mathrm{P}<0.01,{ }^{* * *} \mathrm{P}<0.001 \mathrm{RT}-\mathrm{qPCR}$, reverse transcription-quantitative PCR; miR-27a, microRNA-27a; NC, negative control.

miRNAs have been shown to serve critical roles in a variety of biological and pathological pathways (24). It has been reported that abnormal expression of miRNAs is involved in pregnancy-associated disorders, including PE $(36,37)$. 
miR-136 is highly expressed in decidua-derived mesenchymal stem cells from patients with PE and impairs capillary formation at the maternal-fetal interface by suppressing vascular endothelial growth factor (38). Another previous study indicated that increased miR-34a expression levels promoted trophoblast apoptosis via targeting BCL-2 in PE (39). miR-27a has been studied in number of disease types (36). It has been previously demonstrated that miR-27a and miR-27b regulate the expression of PTEN-induced kinase 1, which is critical for the selective autophagic clearance of damaged mitochondria in Parkinson's disease (40). In maternal diabetes, oxidative stress upregulates miR-27a, which in turn suppresses nuclear factor erythroid 2-related factor 2, and this feedback results in diabetic embryopathy (41). In lung cancer, miR-27a functions as an oncogene contributing to cancer cell proliferation and invasion by targeting SMAD2 and SMAD4 (21). Similar studies have indicated that miR-27a is involved in obesity, gastric cancer chemoresistance and osteosarcoma $(32,42,43)$. However, the roles of miR-27a in PE pathogenesis have not yet been elucidated. In the present study, miR-27a was highly expressed in PE placenta and serum, and contributed to the migration and invasion of trophoblasts, suggesting that miR-27a, at least in part, is involved in PE progression.

SMAD2 belongs to the SMAD family (44). SMAD proteins are signal transducers and transcriptional modulators that mediate multiple signaling pathways (44). Similar to other SMADs, SMAD2 serves a role in the transmission of extracellular signals from TGF- $\beta$ ligands into the cell nucleus and mediates cell proliferation, apoptosis and differentiation (45). The TGF- $\beta$-SMAD2/3 signaling pathway is involved in epithelial-mesenchymal transition in several malignant tumors, including hepatocellular carcinoma (46), breast cancer (47) and colorectal cancer (48). In addition, SMAD2 also contributes to PE initiation (49). TGF- $\beta 1$ suppresses trophoblast invasion by increasing the expression level of connective tissue growth factor mediated by SMAD2/3 (49). Given the significance of miR-27a and SMAD2 in cell invasion, the present study investigated the relationship between them and identified that the anti-invasion function of miR-27a in human trophoblasts in PE partially targets SMAD2.

In conclusion, the present results indicated that miR-27a promoted apoptosis and inhibited proliferation, migration and invasion in human trophoblasts. However, the present study has some limitations, such as the association between the miR-27a expression level and blood pressure or placenta weight was not investigated. Additionally, primary trophoblasts and other human trophoblast cell lines should be included in future studies. Moreover, sample size should be increased in future studies to obtain further evidence. However, the present results indicated that miR-27a may be a promising novel therapeutic target for PE.

\section{Acknowledgements}

Not applicable.

\section{Funding}

This study was supported by Medical and health research projects of Yichang (grant no. A19-301-28).

\section{Availability of data and materials}

The datasets used and/or analyzed during the present study are available from the corresponding author on reasonable request.

\section{Authors' contributions}

WFZ performed experiments and drafted the manuscript. AHC and HJY analyzed data and reviewed the manuscript. LH designed the study and reviewed the manuscript. All authors read and approved the final manuscript.

\section{Ethics approval and consent to participate}

The study was approved by The Ethics Committee of Renmin Hospital of Wuhan University. Written informed consents were collected from all patients.

\section{Patient consent for publication}

Not applicable.

\section{Competing interests}

The authors declare that they have no competing interests.

\section{References}

1. Ghulmiyyah L and Sibai B: Maternal mortality from preeclampsia/eclampsia. Semin Perinatol 36: 56-59, 2012.

2. Mol BWJ, Roberts CT, Thangaratinam S, Magee LA, de Groot CJM and Hofmeyr GJ: Pre-eclampsia. Lancet 387: 999-1011, 2016.

3. Tenorio MB,Ferreira RC, Moura FA, Bueno NB, de Oliveira ACM and Goulart MOF: Cross-talk between oxidative stress and inflammation in preeclampsia. Oxid Med Cell Longev 2019: $8238727,2019$.

4. Sircar M, Thadhani R and Karumanchi SA: Pathogenesis of preeclampsia. Curr Opin Nephrol Hypertens 24: 131-138, 2015.

5. Bhorat I: Pre-eclampsia and the foetus: A cardiovascular perspective. Cardiovasc J Afr 29: 387-393, 2018.

6. Staun-Ram E and Shalev E: Human trophoblast function during the implantation process. Reprod Biol Endocrinol 3: $56,2005$.

7. Rebahi H, Elizabeth Still M, Faouzi Y and Rhassane El Adib A: Risk factors for eclampsia in pregnant women with preeclampsia and positive neurosensory signs. Turk J Obstet Gynecol 15: 227-234, 2018.

8. Xu T, Ni MM, Xing-LI, Li XF, Meng XM, Huang C and Li J: NLRC5 regulates TGF- $\beta 1$-induced proliferation and activation of hepatic stellate cells during hepatic fibrosis. Int J Biochem Cell Biol 70: 92-104, 2016.

9. Miyazono K, Suzuki H and Imamura T: Regulation of TGF-beta signaling and its roles in progression of tumors. Cancer Sci 94: 230-234, 2003.

10. Kawamoto K, Pahuja A, Hering BJ and Bansal-Pakala P: Transforming growth factor beta 1 (TGF-betal) and rapamycin synergize to effectively suppress human $\mathrm{T}$ cell responses via upregulation of FoxP3+ Tregs. Transpl Immunol 23: 28-33, 2010.

11. Serban AI, Stanca L, Geicu OI, Munteanu MC and Dinischiotu A: RAGE and TGF- $\beta 1$ cross-talk regulate extracellular matrix turnover and cytokine synthesis in AGEs exposed fibroblast cells. PLoS One 11: e0152376, 2016.

12. Seoane J and Gomis RR: TGF- $\beta$ family signaling in tumor suppression and cancer progression. Cold Spring Harb Perspect Biol 9: a022277, 2017.

13. Katz LH, Likhter M, Jogunoori W, Belkin M, Ohshiro K and Mishra L: TGF- $\beta$ signaling in liver and gastrointestinal cancers. Cancer Lett 379: 166-172, 2016. 
14. Zhang L, Zhou F, Garcia de Vinuesa A, de Kruijf EM, Mesker WE, Hui L, Drabsch Y, Li Y, Bauer A, Rousseau A, et al: TRAF4 promotes TGF- $\beta$ receptor signaling and drives breast cancer metastasis. Mol Cell 51: 559-572, 2013.

15. Lash GE, Otun HA, Innes BA, Bulmer JN, Searle RF and Robson SC: Inhibition of trophoblast cell invasion by TGFB1, 2 , and 3 is associated with a decrease in active proteases. Biol Reprod 73: 374-381, 2005.

16. Zhao MR, Qiu W, Li YX, Zhang ZB, Li D and Wang YL: Dual effect of transforming growth factor betal on cell adhesion and invasion in human placenta trophoblast cells. Reproduction 132: 333-341, 2006

17. Albers RE, Selesniemi K, Natale DRC and Brown TL: TGF- $\beta$ induces Smad2 phosphorylation, ARE induction, and trophoblast differentiation. Int J Stem Cells 11: 111-120, 2018.

18. Attisano L and Wrana JL: Signal transduction by the TGF-beta superfamily. Science 296: 1646-1647, 2002.

19. Huang Z, Li S, Fan W and Ma Q: Transforming growth factor $\beta 1$ promotes invasion of human JEG-3 trophoblast cells via TGF- $\beta$ /Smad3 signaling pathway. Oncotarget 8: 33560-33570, 2017

20. Zhang J, Zhang X, Xie F, Zhang Z, van Dam H, Zhang L and Zhou F: The regulation of TGF- $\beta$ /SMAD signaling by protein deubiquitination. Protein Cell 5: 503-517, 2014.

21. Chae DK, Ban E, Yoo YS, Kim EE, Baik JH and Song EJ: MIR-27a regulates the TGF- $\beta$ signaling pathway by targeting SMAD2 and SMAD4 in lung cancer. Mol Carcinog 56: 1992-1998, 2017.

22. Marmol I, Sanchez-de-Diego C, Pradilla Dieste A, Cerrada E and Rodriguez Yoldi MJ: Colorectal carcinoma: A general overview and future perspectives in colorectal cancer. Int J Mol Sci 18 E197, 2017.

23. Tang YN, Ding WQ, Guo XJ, Yuan XW, Wang DM and Song JG: Epigenetic regulation of $\mathrm{Smad} 2$ and $\mathrm{Smad} 3$ by profilin-2 promotes lung cancer growth and metastasis. Nat Commun 6 : 8230, 2015.

24. Bartel DP: MicroRNAs: Genomics, biogenesis, mechanism, and function. Cell 116: 281-297, 2004

25. Guo L, Liu Y, Guo Y, Yang Y and Chen B: MicroRNA-423-5p inhibits the progression of trophoblast cells via targeting IGF2BP1. Placenta 74: 1-8, 2018

26. Osipov ID, Zaporozhchenko IA, Bondar AA, Zaripov MM Voytsitskiy VE, Vlassov VV, Laktionov PP and Morozkin ES Cell-free miRNA-141 and miRNA-205 as prostate cancer biomarkers. Adv Exp Med Biol 924: 9-12, 2016.

27. Peng G, Liao Y and Shen C: miRNA-429 inhibits astrocytoma proliferation and invasion by targeting BMI1. Pathol Oncol Res 23: 369-376, 2017.

28. Vaiman D: Genes, epigenetics and miRNA regulation in the placenta. Placenta 52: 127-133, 2017

29. Li P, Guo W, Du L, Zhao J, Wang Y, Liu L, Hu Y and Hou Y: microRNA-29b contributes to pre-eclampsia through its effects on apoptosis, invasion and angiogenesis of trophoblast cells. Clin Sci (Lond) 124: 27-40, 2013.

30. Niu ZR, Han T, Sun XL, Luan LX, Gou WL and Zhu XM: MicroRNA-30a-3p is overexpressed in the placentas of patients with preeclampsia and affects trophoblast invasion and apoptosis by its effects on IGF-1. Am J Obstet Gynecol 218: 249.e1-249.e12, 2018.

31. Fu G, Ye G, Nadeem L, Ji L, Manchanda T, Wang Y,Zhao Y, Qiao J, Wang YL, Lye S, et al: MicroRNA-376c impairs transforming growth factor- $\beta$ and nodal signaling to promote trophoblast cell proliferation and invasion. Hypertension 61: 864-872, 2013.

32. Salah Z, Arafeh R, Maximov V, Galasso M, Khawaled S, Abou-Sharieha S, Volinia S, Jones KB, Croce CM and Aqeilan RI: miR-27a and miR-27a* contribute to metastatic properties of osteosarcoma cells. Oncotarget 6: 4920-4935, 2015.
33. Song J, Li Y and An RF: Identification of early-onset preeclampsia-related genes and MicroRNAs by bioinformatics approaches. Reprod Sci 22: 954-963, 2015.

34. Yang S, Li H, Ge Q, Guo L and Chen F: Deregulated microRNA species in the plasma and placenta of patients with preeclampsia. Mol Med Rep 12: 527-534, 2015.

35. Livak KJ and Schmittgen TD: Analysis of relative gene expression data using real-time quantitative PCR and the 2(-Delta Delta C(T)) method. Methods 25: 402-408, 2001.

36. Jairajpuri DS and Almawi WY: MicroRNA expression pattern in pre-eclampsia (Review). Mol Med Rep 13: 2351-2358, 2016.

37. Pineles BL, Romero R, Montenegro D, Tarca AL, Han YM, Kim YM, Draghici S, Espinoza J, Kusanovic JP, Mittal P, et al: Distinct subsets of microRNAs are expressed differentially in the human placentas of patients with preeclampsia. Am J Obstet Gynecol 196: 261.e1-6, 2007.

38. Ji L, Zhang L, Li Y, Guo L, Cao N, Bai Z, Song Y, Xu Z, Zhang J, Liu $\mathrm{C}$ and Ma X: MiR-136 contributes to pre-eclampsia through its effects on apoptosis and angiogenesis of mesenchymal stem cells. Placenta 50: 102-109, 2017

39. Guo M, Zhao X, Yuan X and Li P: Elevated microRNA-34a contributes to trophoblast cell apoptosis in preeclampsia by targeting BCL-2. J Hum Hypertens 31: 815-820, 2017.

40. Kim J, Fiesel FC, Belmonte KC, Hudec R, Wang WX, Kim C, Nelson PT, Springer W and Kim J: miR-27a and miR-27b regulate autophagic clearance of damaged mitochondria by targeting PTEN-induced putative kinase 1 (PINK1). Mol Neurodegener 11: $55,2016$.

41. Zhao Y, Dong D, Reece EA, Wang AR and Yang P: Oxidative stress-induced miR-27a targets the redox gene nuclear factor erythroid 2-related factor 2 in diabetic embryopathy. Am J Obstet Gynecol 218: 136.e1-136.e10, 2018.

42. Yao F, Yu Y, Feng L, Li J, Zhang M, Lan X, Yan X, Liu Y, Guan F, Zhang $M$ and Chen L: Adipogenic miR-27a in adipose tissue upregulates macrophage activation via inhibiting PPARgamma of insulin resistance induced by high-fat diet-associated obesity. Exp Cell Res 355: 105-112, 2017.

43. Danza K, Silvestris N, Simone G, Signorile M, Saragoni L, Brunetti O, Monti M, Mazzotta A, De Summa S, Mangia A and Tommasi S: Role of miR-27a, miR-181a and miR-20b in gastric cancer hypoxia-induced chemoresistance. Cancer Biol Ther 17: 400-406, 2016.

44. DerynckRandZhang YE: Smad-dependent andSmad-independent pathways in TGF-beta family signalling. Nature 425: 577-584, 2003.

45. Khalil H, Kanisicak O, Prasad V, Correll RN, Fu X, Schips T, Vagnozzi RJ, Liu R, Huynh T, Lee SJ, et al: Fibroblast-specific TGF- $\beta$-Smad $2 / 3$ signaling underlies cardiac fibrosis. J Clin Invest 127: 3770-3783, 2017.

46. Fu H, He Y, Qi L, Chen L, Luo Y, Chen L, Li Y, Zhang N and Guo H: cPLA2 $\alpha$ activates PI3K/AKT and inhibits Smad2/3 during epithelial-mesenchymal transition of hepatocellular carcinoma cells. Cancer Lett 403: 260-270, 2017.

47. Lu Y, Wang L, Li H, Li Y, Ruan Y, Lin D, Yang M, Jin X, Guo Y, Zhang $X$ and Quan C: SMAD2 inactivation inhibits CLDN6 methylation to suppress migration and invasion of breast cancer cells. Int J Mol Sci 18: E1863, 2017.

48. Fleming NI, Jorissen RN, Mouradov D, Christie M, Sakthianandeswaren A, Palmieri M, Day F, Li S, Tsui C, Lipton L, et al: SMAD2, SMAD3 and SMAD4 mutations in colorectal cancer. Cancer Res 73: 725-735, 2013.

49. Cheng JC, Chang HM and Leung PCK: TGF- $\beta 1$ inhibits human trophoblast cell invasion by upregulating connective tissue growth factor expression. Endocrinology 158: 3620-3628, 2017. 